\title{
Capsaicin promotes the development of burst-forming units-erythroid (BFU-E) from mouse bone marrow cells
}

\author{
Seong-Ae Lee ${ }^{1}$, Young-Shin Ryu ${ }^{1}$, \\ Hyung-Im Choi ${ }^{1}$ and In-Seob Han ${ }^{1,2}$ \\ ${ }^{1}$ Immunomodulation Research Center \\ Department of Biological Science \\ University of Ulsan \\ Ulsan 680-749, Korea \\ ${ }^{2}$ Corresponding author: Tel, 82-52-259-2352; \\ Fax, 82-52-259-1694; E-mail, hanis@ulsan.ac.kr
}

Accepted 13 March 2007

Abbreviations: BFU-E, burst-forming units-erythroid; CFU-GM, colony forming units-granulocyte/macrophage; EpoR, erythropoietin receptor; GPA, surface markers glycophorin A; HSC, hematopoietic stem cell

\begin{abstract}
Capsaicin, the pungent component of chilli peppers, is known to induce mediators of hematopoiesis. We investigated the effect of capsaicin on hematopoiesis in mouse progenitor cells. Treatment of mouse bone marrow cells with capsaicin induced the formation of colony of burst-forming units-erythroid (BFU-E). We also found that the number of erythropoietin receptor (EpoR)-positive cells was increased by capsaicin. To clarify the effect of capsaicin on erythroid lineage, BFU-E colonies were separated from non-BFU-E colonies by colonypicking after in vitro culture of mouse bone marrow cells. Quantitative RT-PCR analysis revealed that capsaicin stimulated the expression of the erythroid-specific genes encoding EpoR, glycophorin A (GPA), $\beta$-globin (Hbb-b1), GATA-1, PU.1, nuclear factor erythroid-derived 2 (NF-E2), and Krüppel-like factor 1 (KLF1) in the BFU-E colonies. Furthermore, capsaicin could effectively stimulate the transfected GATA-1 promoter in K562 cells. GATA-1 is known as an essential transcription factor for the development of erythroid cells. Our results show that development of the erythroid lineage from bone marrow cells can be induced by treatment with capsaicin, and that GATA-1 seems to play a role in this induced erythroid maturation.
\end{abstract}

Keywords: capsaicin; erythroid progenitor cells; erythropoiesis; GATA1 transcription factor; receptors, erythropoietin

\section{Introduction}

Hematopoiesis is a paradigmatic development system, representing the progressive restriction of the cell fate potential of initially multipotent hematopoietic stem cells (HSCs). The developmental pathways from HSCs to mature cells contain many lineage branch points, and once lineage choices are made in response to physiological needs, progenitors display increasingly limited lineage potentials. Lineage commitment of HSCs involves the upregulation of particular transcription programs and the concomitant suppression of multipotentiality and transcriptional programs associated with other lineages. During hematopoiesis, cytokines act as developmental signals that orchestrate the commitment of progenitor cells into and through particular lineages. The differentiation of HSCs to erythroid cells is controlled by a complex array of extrinsic and intrinsic factors, but the detailed mechanisms involved remain poorly defined; GATA-1, erythropoietin receptor (EpoR), surface markers glycophorin $A$ (GPA), and $\beta$-globin mRNA are up-regulated, whereas GATA-2 mRNA is down-regulated (Edvardsson et al., 2004).

Capsaicin (trans-8-methyl- $N$-vanillyl-6-nonenamide) is the major phenolic pungent principle of hot peppers of the genus Capsicum. It has been used against neuropathic pain and inflammation, and as an anti-carcinogen (Winter et al., 1995; Kim et al., 2003; Lee and Park, 2003), suggesting that it may be an important dietary phytochemical with potential therapeutic activities (Surh et al., 1998). A recent study revealed that capsaicin attenuated the transcription of cytokines, GM-CSF, IFN- $\gamma$, and IL-2 (Gertsch et al., 2002). In addition, capsaicin administration enhanced the release of calcitonin gene-related peptide (CGRP) and substance $\mathrm{P}$ (Katagiri et al., 2005; Mizuguchi et al., 2005), which stimulates colony forming units-granulocyte/macrophage (CFU-GM) colony formation.

The goal of the present study was to explore the effect of capsaicin on the development of the erythroid lineage in bone marrow HSCs in vitro. 


\section{Materials and Methods}

\section{Cells and cell culture}

Bone marrow cells were obtained from 5-week-old male C57BL/6 mice and separated from dead cells and RBCs using Lympholyte M (Cedarlane Laboratories Ltd. Mornby, Canada). Cells were incubated in Iscove's modified Dulbecco's medium (IMDM) containing $15 \%$ FBS, $1 \% \mathrm{BSA}, 10 \mathrm{mg} / \mathrm{ml}$ transferrin, 100 $\mu \mathrm{M}$ 2-mercaptoethanol, $2 \mathrm{mM}$ L-glutamine, and $3 \mathrm{U} /$ $\mathrm{ml}$ of erythropoietin (M3334; StemCell Technologies, Vancouver, Canada). Cells were plated in $35-\mathrm{mm}$ culture dishes. K562 (chronic myelogenous leukemia) cells were cultured in RPMI medium containing $10 \%$ (v/v) FBS (Yang et al., 2006). Before transfection and treatment, the cells were kept in medium supplemented with $0.1 \%$ FBS for $24 \mathrm{~h}$.

\section{Colony-forming cell assays}

In vitro colony-forming assays were carried out in triplicate by plating $3 \times 10^{5} \mathrm{cell} / \mathrm{ml}$ in M3334 with capsaicin (1, 10, and $100 \mathrm{pM}$ ) and counting on 8 day culture. Capsaicin (Sigma, St. Louis, MO) was dissolved in DMSO (Sigma) and diluted in culture medium before treatment. Colonies were counted under an inverted microscope as previously described (Han et al., 2003).

\section{Flow cytometry}

FACS analysis was performed using a Becton Dickinson FACS Calibur (Oxnard, CA). Colonies were harvested after $8 \mathrm{~d}$, and blocked with $2.4 \mathrm{G} 2$ antiFc $\gamma R$ antibody (BD PharMingen, San Diego, CA) for $10 \mathrm{~min}$ at $4^{\circ} \mathrm{C}$. The cells were washed twice with FACS buffer $\left(0.1 \% \mathrm{BSA}, 0.02 \% \mathrm{NaN}_{3}\right.$ in $\left.1 \times \mathrm{PBS}\right)$ and stained with anti-EpoR antibody (Santa Cruz Biotechnology Inc., Santa Cruz, CA), followed by FITC-conjugated anti-rabbit antibodies (Jackson Immunoresearch, West Grove, PA).

\section{RT-PCR analysis}

Burst-forming units-erythroid (BFU-E) and non BFU$E$ cells (day 8 ) were picked with Pasteur pipettes. The cells were washed with $1 \times$ PBS and total RNA was prepared using Tri-reagent (Sigma). Total RNA was reverse-transcribed with oligo (dT)18 primer (Invitrogen, Carlsbad, CA) using RevertAid ${ }^{\mathrm{TM}}$ M-MuLV Reverse Transcriptase (Fermentas Life Sciences, Hanover, MD) and PCR reactions were performed. The amount of cDNA was normalized using the housekeeping gene, GAPDH. Cycling parameters were denaturation at $94^{\circ} \mathrm{C}$ for $15 \mathrm{~s}$, annealing at $60^{\circ} \mathrm{C}$ for $30 \mathrm{~s}$, and extension at $72^{\circ} \mathrm{C}$ for $30 \mathrm{~s}$. The primer sets were as follows: GAPDH, sense primer 5'-GCA-
TTGTGGAAGGGCTCATG-3' and reverse primer 5'GATGCCTGCTTCACCACCTT-3'; EpoR, sense primer 5'-TCCTGGAGCACCTATGACCA-3' and reverse primer 5'-ACCGTCAGCAACAGCGAGAT-3'; GPA, sense primer 5'-CTGTGGTGGCTTCAACTGTA-3' and reverse primer 5'-CGATAATCCCTGCCATCACG-3'; GATA-1, sense primer 5'-CACTTCCCCAAATGCAGCCA-3' and reverse primer 5'-GAAAGGACTGGGAAAGTCAG-3'; GATA-2, sense primer 5'-GCCGTATTGAATGCGCAGCA-3' and reverse primer 5'-AGCCCCTGGGTAAACAGACA-3'; PU.1, sense primer 5'-TGACTACTACTCCTTCGTGG-3' and reverse primer 5'-GATAAGGGAAGCACATCCGG-3'; nuclear factor erythroid-derived 2 (NF-E2), sense primer 5'-TGTTGGGGAGCTTGGAGAGA-3' and reverse primer 5'-TGAGCAGGCCTGAAAGGGTA-3'; Krüppel-like factor 1 (KLF1), sense primer 5'- CCATCAGTACACTAACCACC-3' and reverse primer 5'-ACAGGTCACGTCCCTCTCAT-3'; $\beta$-globin (Hbb-b1), sense primer 5'- AAGGCACCTTTGCCAGCCTCA-3' and reverse primer 5'-CGTTAGTGGTACTTGTGAGCC-3'.

\section{Real-time PCR}

Real-time PCR was performed using SYBR green I Taq polymerase (Qiagen, Hilden, Germany) according to the manufacturer's protocol with an MJ Research Opticon ${ }^{T M}$ Continuous Fluorescence Detector. All assays were carried out twice as independent PCR runs for each cDNA sample. Threshold cycles (CT) were determined with Opticon Monitor $^{\mathrm{TM}}$ analysis software, and used to calculate the relative amount of each transcript.

\section{Transient transfection}

Murine genomic DNA was extracted from C57BL/6 mouse splenocytes by standard procedures. Reporter assay plasmids were constructed with upstream fragments of $1041 \mathrm{bp}$ and $912 \mathrm{bp}$ of mouse GATA-1 (mGATA-1) and GATA-2 (mGATA-2) genes, respectively, based on the sequences in GenBank (Accession Nos X57530 and NT039353). Transfection of the mGATA-1 or mGATA-2 reporter constructs in pGL3-basic (Promega, Madison, WI), or of empty pGL3-basic, into K562 cells was performed with Lipofectamine ${ }^{\mathrm{TM}}$ (Invitrogen). For each experiment, exponential K562 cells (4 × $10^{4}$ cells/well) were cotransfected with $0.8 \mu \mathrm{g}$ of pGL3GATA-1 or pGL3-GATA-2 and $40 \mathrm{ng}$ of pCMV- $\beta$ Gal per well. After $24 \mathrm{~h}$, capsaicin $(0.1$ and $1 \mu \mathrm{M})$ was added and the cells were cultured for an additional $24 \mathrm{~h}$. Cell lysates were prepared in lysis buffer (Promega) and luciferase activity was measured using a Wallac Multichannel Counter C (Perkin Elmer Inc., Gathisburg, MD). Ratios of arbitrary 
luciferase light units to $\beta$-galactosidase activities were calculated and compared to those of cells transfected with empty pGL3-basic. All results are presented as fold increases or decreases over the standardized luciferase activity in the absence of capsaicin.

\section{Results}

\section{Capsaicin increases erythroid lineage development} in mouse bone marrow cells cultured in vitro

To investigate the effects of capsaicin on lineage development in mouse HSC, bone marrow cells were exposed to a range of capsaicin concentrations, and colony formation was monitored. As

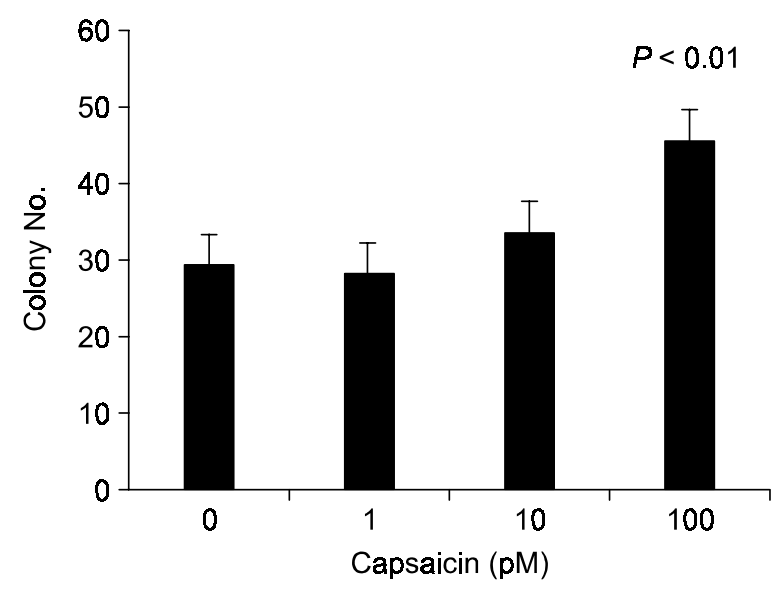

Figure 1. Effect of capsaicin on erythroid lineage development from mouse bone marrow cells. Mouse (C57BL/6 male 5 weeks) bone marrow cells were cultured in M3334 methylcellulose medium in the presence of the indicated concentrations of capsaicin. Morphology of colonies on day 8 was assessed by the hematopoietic progenitor cell colony-forming assay. Results of triplicate experiments are shown as means $\pm S E$ of the number of colony formation. shown in Figure 1, treatment with 100 pM capsaicin enhanced the number of BFU-E colonies 1.5-fold. We carried out FACS analysis of the bone marrow cells stained with anti-EpoR antibody to determine whether capsaicin affected expression of this erythroid lineage marker. Treatment with capsaicin increased erythroid differentiation, as measured by the fraction of cells expressing EpoR (Figure 2).

\section{Capsaicin induces the expression of erythropoiesis- associated genes in BFU-E colonies}

Since whole cells cultured from bone marrow include several lineages, we focused on the influence of capsaicin on the development of erythroid progenitor cells. After in-vitro culture of mouse bone marrow cells on methylcellulose medium, BFU-E colonies and non BFU-E colonies were picked separately under a microscope and total RNAs were obtained from the two cell types. We performed three independent experiments evaluating the expression of erythropoiesis-related genes in these cells by RT-PCR. When colonies were harvested on 8 day culture, capsaicin increased EpoR and GPA mRNAs in the BFU-E cells and there was no significant change in the non-BFU-E cells (Figure 3). EpoR mRNA, which was barely expressed in quiescent hematopoietic progenitor cells (HPCs), is slowly induced in the erythropoietic pathway, whereas it is hardly expressed in the granulopoietic pathway (Labbaye et al., 1995). GATA-1 expression was increased 4-fold by capsaicin in the BFU-E cells, whereas capsaicin did not affect GATA-2 expression in either the BFU-E or non-BFU-E cells. In addition, PU.1 and $\beta$-globin transcripts increased in the BFU-E cells with no increase in the non-BFU-E cells. NF-E2 and KLF1 mRNAs, which are constantly expressed in the erythroid pathway, were highly induced in the BFU-E cells.

To examine whether capsaicin can stimulate the expression of erythropoiesis-related genes in the
A

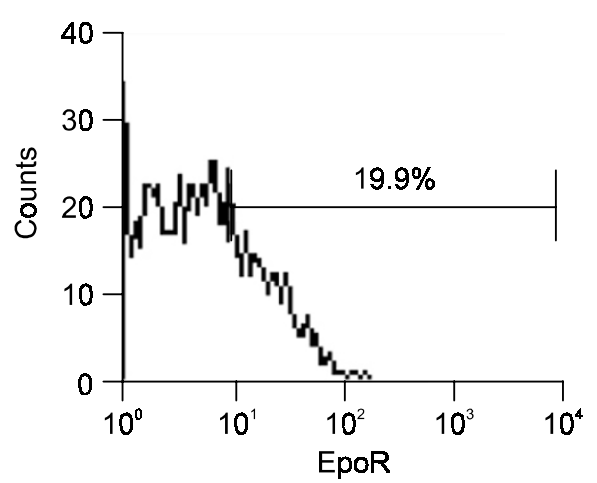

B

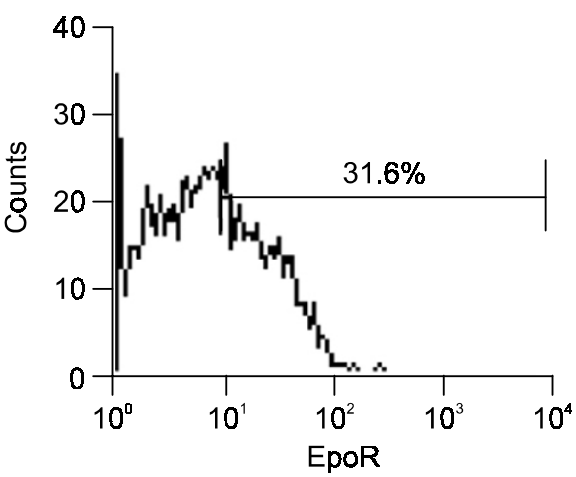

Figure 2. Flow cytometric analysis of EpoR-expression in mouse bone marrow cells. Mouse bone marrow cells were cultured in the presence of capsaicin for $8 d$ and colonies were harvested for flow cytometric analysis. The graphs show cell numbers versus highly fluorescent FITC EpoR-expressing cells. (A) mock; (B) 10 pM capsaicin. 
A

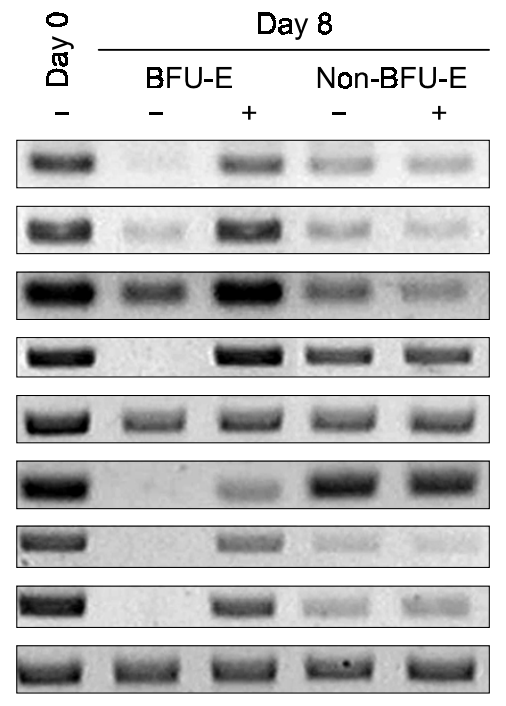

B

Capsaicin
EpoR
GPA
Hbb-b1
GATA-1
GATA-2
PU.1
NF-E2
KLF1
GAPDH
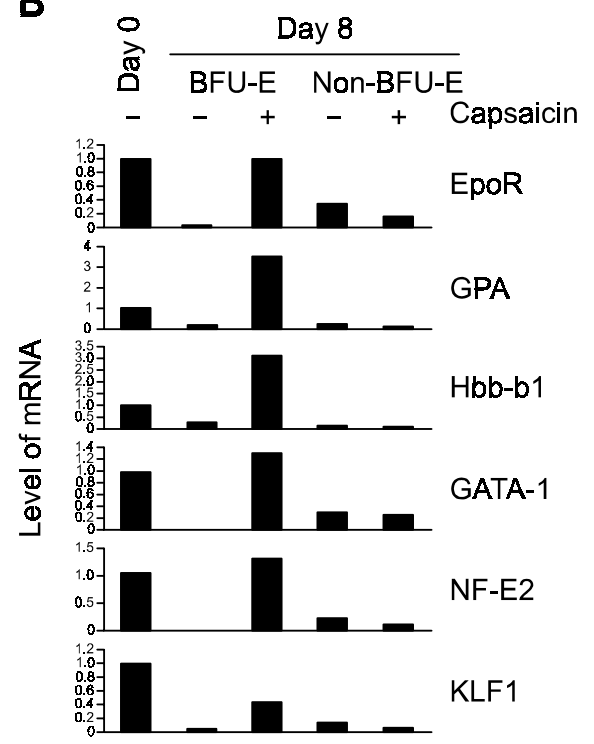

C

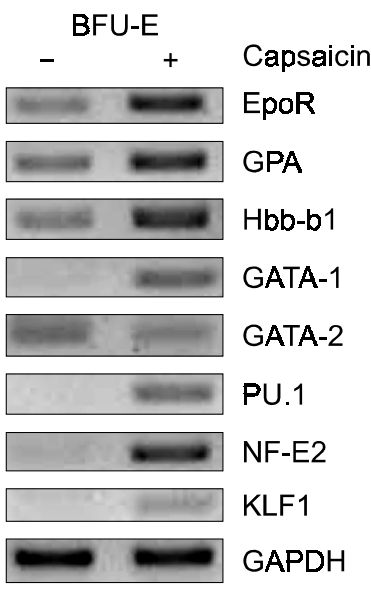

Figure 3. Expression of EpoR, GPA, GATA-1, PU.1, Hbb-b1, NF-E2, KLF1 and GATA-2 in BFU-E cells treated with capsaicin. RNA isolated from the BFU-E cells after culture on M3334 medium was subjected to reverse transcription. (A) RT-PCR and (B) Quantitative real-time PCR were performed and the data were normalized to GAPDH. (C) Capsaicin solution (1 $\mu \mathrm{M}$ of $10 \mathrm{pM} /$ colony) was dropped directly onto the colonies and after $2 \mathrm{~d}$, expression of genes in the colonies was analyzed by RT-PCR.

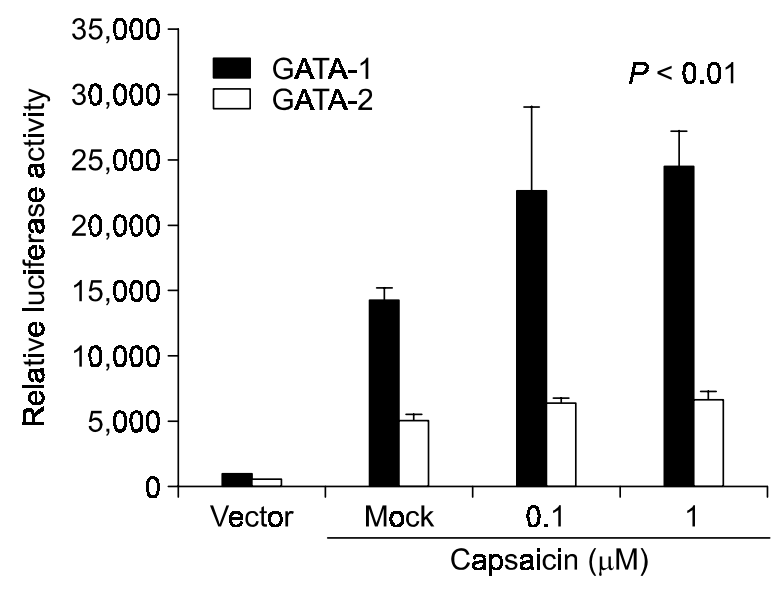

Figure 4. Induction of luciferase activity driven by the GATA-1 promoter by capsaicin. K562 cells were transfected with constructs (pGL3-GATA-1, pGL3-GATA-2 or empty pGL3-basic as a control). After transfection, cells were incubated with or without capsaicin $(0,0.1,1$ $\mu \mathrm{M}$ of final concentration) for $24 \mathrm{~h}$ and luciferase and $\beta$-galactosidase activities were measured. Results represent luciferase/ $\beta$-galactosidase activities. Triplicates were used for each experiment and results are shown as means \pm SE.

differentiated BFU-E cells, we administrated capsaicin directly to the BFU-E colonies on day 6 and assessed expression of erythropoiesis-associated genes in individual colonies by RT-PCR analysis on day 8 (Figure $3 \mathrm{C}$ ). The expression pattern of genes was consistent with the one shown in Figure $3 \mathrm{~A}$.
Activation of the GATA-1 promoter by capsaicin To ascertain the effect of capsaicin on GATA-1 promoter activity, K562 cells were transiently transfected with various reporter constructs. Our results showed that the luciferase activity driven by the GATA-1 promoter was increased by capsaicin, whereas GATA-2 promoter activity was not affected (Figure 4).

\section{Discussion}

Red blood cell development starts with the commitment of pluripotent hematopoietic stem cells into self-renewing lineage specific progenitors, including BFU-E and CFU-E. A key issue in HSC studies has focused on how their commitment to a particular lineage and maturation is regulated at the cellular and molecular levels. This issue is of great clinical relevance (Goodnough and Marcus,1998; Beauchemin et al., 2004; Kostaridou et al., 2004; Chung et al., 2005).

We have shown here that capsaicin can act as an inducer of bone marrow erythropoiesis. Capsaicin treatment of mouse bone marrow cells resulted in an increase in formation of BFU-E colonies and upregulation of an erythroid marker, EpoR. However, bone marrow cells are fairly heterogeneous in lineage and development stage. Therefore, it was necessary to further separate the cells before 
analyzing the erythroid-associated gene expression pattern. BFU-E colonies were collected for RT-PCR analysis, and the induction of erythroid-specific gene transcription by capsaicin was detected both in the BFU-E colonies and in the whole bone marrow experiments. The induction of erythroid-specific gene transcription by capsaicin was also observed by an experiment in which capsaicin solution was dropped onto BFU-E colonies and transcripts levels were analyzed. This result indicates that capsaicin can stimulate expression of erythroid-specific gene even after commitment to erythroid progenitor cells. We found that transcription of the erythroid markers, GPA and EpoR, was strongly induced by capsaicin. GPA has been used to characterize erythroid differentiation because its expression is observed exclusively in erythroid cultures and parallels the expression of $\beta$-globin. Signaling through EpoR regulates the proliferation, differentiation, and survival of erythroid progenitor cells (Lu et al., 2000). During erythrocyte differentiation cells became progressively more sensitive to erythropoietin due to the production of EpoR in these cells. Therefore, our results underscore the likelihood that capsaicin promotes the erythroid pathway by acting upstream of the Epo/EpoR-mediated regulation of erythroid differentiation or proliferation. The different differentiation potentials of cells at the progenitor stage may reflect their differential expression of specific combinations of lineage-related transcription factors. Our data support the notion that capsaicin may promote erythroid lineage commitment by regulating the expression of transcription factors such as GATA-1 and NF-E2. In addition, transient transfection assays showed that capsaicin stimulated the expression of a GATA-1 reporter in K562 cells, but not of a GATA-2 reporter. These results support the hypothesis that capsaicin enhances erythroid lineage development by up-regulating GATA-1.

GATA-1 stimulates megakaryocyte/erythroid lineage commitment and simultaneously inhibits granulocyte/monocyte (GM) development (Iwasaki et al., 2003). Studies in mice and in cell lines have pointed to a major role of GATA-1 in erythroid commitment and in the subsequent erythroid differentiation (Cantor and Orkin, 2002). GATA-1 is barely expressed in quiescent erythroid progenitors, but is rapidly induced when these cells are stimulated towards erythroid differentiation by Epo. It has been shown using conditional knockout models that the expression of GATA-1 in embryonic stem cells strongly induces expression of $\mathrm{Bcl}-\mathrm{XL}$, but not $\mathrm{Bcl}-2$, in erythroid cells, and this may be the basis of one of the anti-apoptotic effects of GATA-1 in erythroid cells (Gregory et al., 1999).

We have noted that capsaicin did not change
GATA-2 expression. GATA-2 is highly expressed in HSCs and declines with blood cell maturation, implying that it is a key factor in the maintenance of stem cell function, but not in provoking cell fate decisions in the HSCs. An experiment using haploinsufficiency of GATA-2 in mice indicated that the level of GATA-2 selectively affected GM colony formation (Rodrigues et al., 2005). Down regulation of GATA-2 expression was required for stem cells to contribute to normal hematopoiesis in vivo. Capsaicin may not affect the GATA-2 regulation, which is associated with normal development of the CFU-GM lineage.

The expression of PU.1 is tightly controlled in hematopoietic cells (Rosenbauer et al., 2004). We observed that capsaicin increased PU.1 expression in non-BFU-E colonies, which were mainly myeloidlineage cells, but not in BFU-E colonies. This suggests that capsaicin can stimulate HSC commitment to the myeloid lineage by regulating the PU.1 transcription factor. This is supported by previous evidence that PU.1 prevents differentiation and maintains self-renewal in the erythroid lineage, whereas it is a positive regulator of myeloid-lineage differentiation (Back et al., 2004).

We cannot be certain which signaling pathway(s) is involved in the promotion of the BFU-E lineage by capsaicin. The inhibitory effect of TNF- $\alpha$ on erythroid maturation seems to involve $N F-\kappa B$ induction (Xiao et al., 2002). Later in differentiation, a decline in the levels of the NF- $\mathrm{KB}$ family of factors leads to derepression and a consequent increase of NF-E2, which in turn activates a subset of erythroid-specific genes (Liu et al., 2003). Capsaicin can block NF-kB activation by suppressing the degradation of $I_{\kappa} \mathrm{B} \alpha$ (Singh et al., 1996). These results together suggest that the enhanced erythroid-specific gene expression in response to capsaicin is mediated via the $\mathrm{NF}-\kappa \mathrm{B}$ signaling pathway. This hypothesis is supported by our observation that the level of NF-E2 mRNA is also increased by capsaicin.

\section{Acknowledgement}

This work was supported by the Research Fund of the SRC of the Ministry of Science and Technology of Korea to University of Ulsan IRC. It was supported in part by BK21 program to S.L and H.C.

\section{References}

Back J, Dierich A, Bronn C, Kastner P, Chan S. PU.1 determines the self-renewal capacity of erythroid progenitor cells. Blood 2004;103:3615-23

Beauchemin H, Blouin MJ, Trudel M. Differential regulatory 
and compensatory responses in hematopoiesis/erythropoiesis in alpha- and beta-globin hemizygous mice. J Biol Chem 2004;279:19471-80

Cantor AB, Orkin SH. Transcriptional regulation of erythropoiesis: an affair involving multiple partners. Oncogene 2002;21:3368-76

Chung JW, Kim GY, Mun YC, Ahn JY, Seong CM, Kim JH. Leukotriene B4 pathway regulates the fate of the hematopoietic stem cells. Exp Mol Med 2005;37:45-50

Edvardsson L, Dykes J, Olsson ML, Olofsson T. Clonogenicity, gene expression and phenotype during neutrophil versus erythroid differentiation of cytokine-stimulated CD34+ human marrow cells in vitro. Br J Haematol 2004;127:451-63

Gertsch J, Guttinger M, Sticher O, Heilmann J. Relative quantification of mRNA levels in Jurkat T cells with RT-real time-PCR (RT-rt-PCR): new possibilities for the screening of anti-inflammatory and cytotoxic compounds. Pharm Res 2002;19:1236-43

Goodnough LT, Marcus RE. Erythropoiesis in patients stimulated with erythropoietin: the relevance of storage iron. Vox Sang 1998;75:128-33

Gregory T, Yu C, Ma A, Orkin SH, Blobel GA, Weiss MJ. GATA-1 and erythropoietin cooperate to promote erythroid cell survival by regulating bcl-xL expression. Blood 1999;94:87-96

Han IS, Ra JS, Kim MW, Lee EA, Jun HY, Park SK, Kwon BS. Differentiation of CD34+ cells from human cord blood and murine bone marrow is suppressed by $\mathrm{C} 6$ beta-chemokines. Mol Cells 2003;15:176-80

Iwasaki $\mathrm{H}$, Mizuno $\mathrm{S}$, Wells RA, Cantor $\mathrm{AB}$, Watanabe $\mathrm{S}$, Akashi K. GATA-1 converts lymphoid and myelomonocytic progenitors into the megakaryocyte/erythrocyte lineages. Immunity 2003;19:451-62

Katagiri F, Inoue S, Sato Y, Itoh H, Takeyama M. Ecabet sodium raises plasma levels of calcitonin generelated peptide and substance P in healthy humans. J Pharm Pharmacol 2005; 57:799-805

Kim CS, Kawada T, Kim BS, Han IS, Choe SY, Kurata T, Yu R. Capsaicin exhibits anti-inflammatory property by inhibiting IkB-a degradation in LPS-stimulated peritoneal macrophages. Cell Signal 2003;15:299-306

Kostaridou S, Polychronopoulou S, Premetis E, Papassotiriou I, Stamoulakatou A, Haidas S. Ineffective erythropoiesis underlies the clinical heterogeneity of congenital dyserythropoietic anemia type II (CDA II). Pediatr Int 2004;46:274-9

Labbaye C, Valtieri M, Barberi T, Meccia E, Masella B, Pelosi
E, Condorelli GL, Testa U, Peschle C. Differential expression and functional role of GATA-2, NF-E2, and GATA-1 in normal adult hematopoiesis. J Clin Invest 1995;95:2346-58

Lee BM, Park KK. Beneficial and adverse effects of chemopreventive agents. Mutat Res 2003;523-524:265-78

Liu JJ, Hou SC, Shen CK. Erythroid gene suppression by NF-kappa B. J Biol Chem 2003;278:19534-40

Lu L, Ge Y, Li ZH, Dai MS, Broxmeyer HE. Enhancement of proliferation and differentiation of erythroid progenitors by co-transduction of erythropoietin receptor and $\mathrm{H}$-ras cDNAS into single CD34 (3+) cord blood cells. Bone Marrow Transplant 2000;26:817-22

Mizuguchi S, Ohno T, Hattori Y, Kamata K, Arai K, Saeki T, Saigenji K, Hayashi I, Kuribayashi Y, Majima M. Calcitonin gene-related peptide released by capsaicin suppresses myoelectrical activity of gastric smooth muscle. J Gastroenterol Hepatol 2005;20:611-8

Rodrigues NP, Janzen V, Forkert R, Dombkowski DM, Boyd AS, Orkin SH, Enver T, Vyas P, Scadden DT. Haploinsufficiency of GATA-2 perturbs adult hematopoietic stem-cell homeostasis. Blood 2005;106:477-84

Rosenbauer F, Wagner K, Kutok JL, Iwasaki H, Le Beau MM, Okuno Y, Akashi K, Fiering S, Tenen DG. Acute myeloid leukemia induced by graded reduction of a lineage-specific transcription factor, PU.1. Nat Genet 2004;36:624-30

Singh S, Natarajan K, Aggarwal BB. Capsaicin (8-methyl-Nvanillyl-6-nonenamide) is a potent inhibitor of nuclear transcription factor-kappa B activation by diverse agents. J Immunol 1996;157:4412-20

Surh YJ, Kim SG, Park KK, Sohn Y, Lee JM, Kim ND, Miller JA. Chemopreventive effects of 2-(allylthio)pyrazine on hepatic lesion, mutagenesis and tumorigenesis induced by vinyl carbamate or vinyl carbamate epoxide. Carcinogenesis 1998; 19:1263-7

Winter J, Bevan S, Campbell EA. Capsaicin and pain mechanisms. Br J Anaesth 1995;75:157-68

Xiao W, Koizumi K, Nishio M, Endo T, Osawa M, Fujimoto K, Sato I, Sakai T, Koike T, Sawada K. Tumor necrosis factor-alpha inhibits generation of glycophorin A+ cells by CD34+ cells. Exp Hematol 2002;30:1238-47

Yang $\mathrm{SH}$, Chien CM, Lu MC, Lin YH, Hu XW, Lin SR. Up-regulation of Bax and endonuclease $G$, and downmodulation of $\mathrm{Bcl}-\mathrm{XL}$ involved in cardiotoxin III-induced apoptosis in K562 cells. Exp Mol Med 2006;38:435-44 